\title{
Synthesis and Biological Activity of Novel Statine Derivatives Containing Ferrocenyl Moiety
}

\author{
Lijun Gu, Bingqin Yang* and Feilong Liu \\ Key Laboratory of Synthetic and Natural Functional Molecule Chemistry (Ministry of Education), \\ Department of Chemistry, Northwest University, Xi'an 710069, People's Republic of China
}

\begin{abstract}
$\mathrm{Na}$ busca por novos inibidores de protease aspartica, conjugados de ferroceno com estatina foram planejados e sintetizados através de reação de aclopamento usando o protocolo padrão $N, N$ '-diciclohexilcarboimida (DCC) e 1-hidroxibenzotrizol (HOBt). Os novos compostos foram caracterizados por espectroscopia de IR, ${ }^{1} \mathrm{H}$ NMR, MS e análise elementar. Os resultados do bioensaio mostraram que alguns dos novos compostos podem servir como ponto de partida.
\end{abstract}

In a search for new aspartic protease inhibitors, conjugates of ferrocene with statine were designed and synthesized by coupling reaction using the standard $N, N$ '-dicyclohexylcarbodiimide (DCC) and 1-hydroxybenzotriazole (HOBt) protocol. The title compounds were characterized by IR, ${ }^{1} \mathrm{H}$ NMR spectroscopy, MS and elemental analysis. The results of bioassay showed that some title compounds could serve as a starting point.

Keywords: statine, ferrocene, aspartic protease, synthesis, biological activity

\section{Introduction}

Aspartic proteases are involved in many biological pathways in fungi, plants, humans, parasites, retroviruses. ${ }^{1-2}$ Aspergillus oryzae aspartic proteinase is found to have activity not only for the activation of trypsinogen but also for the activation of chimotrypsinogen with the cleavage of the Arg15-Ile16 bond. ${ }^{3}$ Statine, the $(3 S, 4 S)$-4-amino-3hydroxy-6-methylheptanoic acid configurational isomer is a key component of pepstatin and of other synthetic peptide inhibitors of aspartic protease. ${ }^{4}$ It is well known that long-chain peptides can exhibit poor penetration and are generally unsuitable for a metabolically stable drug, because of enzymatic degradation..$^{5}$ In many case, this inconvenience can be circumvented by shortening the length of the inhibitors.

Ferrocene is a member of a special organometallic group known as metallocenes or "sandwich" molecules. Among metallocenes there are compounds with antiproliferative effect, antibiotics, and inhibitors of enzymes. ${ }^{6-9}$ Conjugates of ferrocene with well-known drugs were reported. ${ }^{10-11}$ Ferrocene-containing compounds often possess unexpected biological activity. ${ }^{12}$ We report here the search for shorter aspartic protease inhibitors resulting from introducing

*e-mail: gulijun2005@126.com ferrocenyl unit into statine and synthesized the novel statine derivatives which might provide interesting biological properties. The structure of target compounds is listed in Table 1.

\section{Experimental}

\section{Materials and equipment}

All the reactions were carried out under a nitrogen atmosphere with the exclusion of moisture. All the amino acids used were L-amino acids. N-Boc-statine and N-BocAHPPA were synthesized according to the method described in the literature. ${ }^{13}$ Ferrocenylmethylamine was synthesized by the literature method of Kelly et al. ${ }^{14}$ For syntheses of compounds $\mathbf{4}$ and $\mathbf{1 0}$ see reference. ${ }^{15}$ Solvents were purified and dried by standard methods. The melting points were determined on an XT-4 micro melting point apparatus and uncorrected. IR spectra were recorded on an EQUINOX-55 spectrometer on a $\mathrm{KBr}$ matrix. ${ }^{1} \mathrm{H}$ NMR spectra were recorded on an INOVA-400 NMR spectrometer using TMS as an internal standard. Chemical shift values $(\delta)$ are given in ppm. Elemental analyses were performed on a Vario EL III CHNS analyzer. Electrospray mass spectra were obtained with an MALDI-TOF Mass spectrometer. 200-300 mesh silica gel was used for column chromatography. 
Table 1 Structure of target comounds and their biological activities

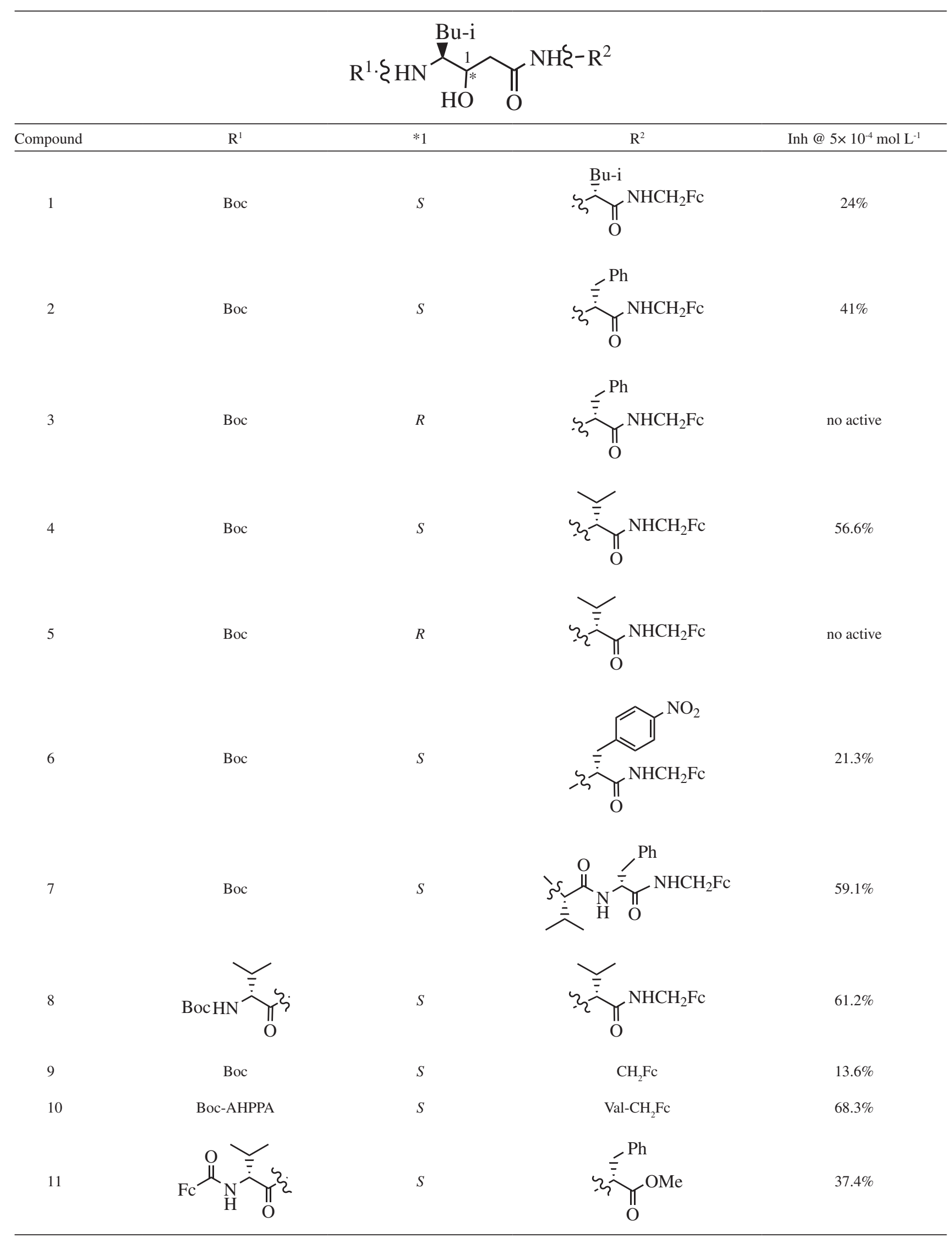


General procedure A: Coupling reactions using DCC / $\mathrm{HOBt}$

The amino compound ( $1 \mathrm{mmol}$ ) was dissolved in DCM (1 mL) and DMF (1 mL). Boc-amino acid (1.25 mmol) and HOBt $(1.4 \mathrm{mmol})$ were added followed by a solution of DCC $(1.4 \mathrm{mmol})$ in DCM $(3 \mathrm{~mL})$. The reaction mixture was allowed to stir at $0{ }^{\circ} \mathrm{C}$ for $5 \mathrm{~h}$ and at room temperature overnight. DCU was filtrated, and the filtrate was evaporated under reduced pressure. The residue was dissolved in ethyl acetate, washed successively with cold $1 \mathrm{~mol} \mathrm{~L}-1 \mathrm{HCl}$, saturated $\mathrm{NaHCO}_{3}$, and saturated $\mathrm{NaCl}$, and dried $\left(\mathrm{MgSO}_{4}\right)$. The peptide was purified by silica gel chromatography ( $3 \%$ methanol in chloroform (v/v)).

\section{General procedure B: Removal of the tert-butoxycarbonyl} group

The Boc-peptide (1 equiv.) in a solution of $4 \mathrm{~mol} \mathrm{~L}^{-1}$ $\mathrm{HCl}$ in dioxane (10 equiv. of $\mathrm{HCl}$ ) was stirred at room temperature and the reaction monitored by TLC. Complete reaction was generally achieved in about $30 \mathrm{~min}$. Excess reagent was removed under reduced pressure to give a solid residue. The residue was washed three times with ethyl ether and dried in vacuum over $\mathrm{KOH}$ and $\mathrm{P}_{2} \mathrm{O}_{5}$ for several hours. The resulting hydrochloride was used without further purification. The hydrochloride was neutralized with NMM in DCM to give free amine.

\section{Bioassay method}

The inhibition of the tested bacteria (Aspergillus oryzae) was determined with the method of dull colony notation. ${ }^{16}$ All compounds tested were dissolved in DMSO $(500 \mu \mathrm{mol})$ and subsequently diluted in the culture medium before treatment of the suspension of the tested bacteria. The suspension at three concentrations was inoculated on the culture medium treated with compound and incubated at room temperature for $60 \mathrm{~h}$. In parallel, the tested bacterial was treated with DMSO as control. For each compound, three repetitions were conducted to ensure the reliability of the results. The percent inhibition was determined using the following relationship: inhibition rate $(\%)=[$ av colony numbers of control (not treated with compound) - av colony numbers smeared with drugs] / av colony numbers without drugs.

\section{Results and Discussion}

The representative synthesis of various inhibitors containing ferrocenyl moiety is outlined in Scheme 1 and 2. Boc-protected leucine $\mathbf{1 3}$ was coupled to aminomethylferrocene 12 using DCC and HOBt as the coupling agent. The Boc-group was removed by treatment with $4 \mathrm{~mol} \mathrm{~L}^{-1} \mathrm{HCl}$ in dioxane, neutralized with $N$-methylmorpholine (NMM), and the resulting free amine was allowed to couple subsequently with N-Boc-statine using the same coupling sequence to give the compound $\mathbf{1}$. Other compounds (such as $\mathbf{5}, \mathbf{7}$, etc.) in Table 1 were prepared by analogous sequence as described above.

Phenylalanine methyl ester hydrochloride $\mathbf{1 6}$ was neutralized with NMM. Using the method described above, compound 11 was obtained in $27.6 \%$ yield for the coupling/ deprotection steps.

The target compounds are characterized by IR and ${ }^{1} \mathrm{H}$ NMR spectroscopy, MALDI-TOF MS and elemental analyses. The results are in accordance with the expected
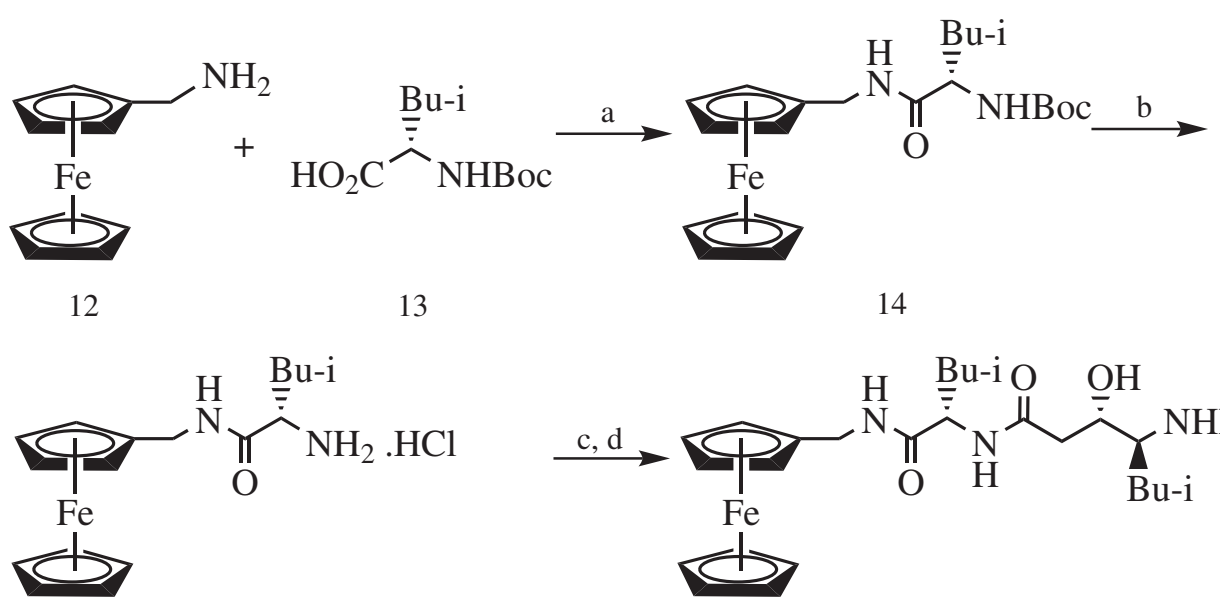

15

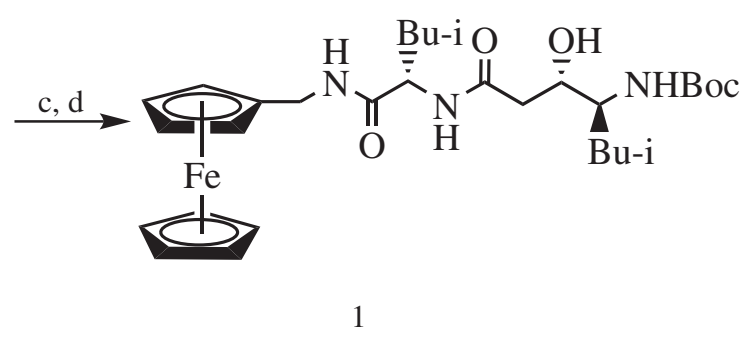

Scheme 1. Synthesis of compound 1. Reagents and conditions: (a) DCC/HOBt; (b) 4 mol L-1 HCl/dioxane; (c) NMM/DCM, (d) N-Boc-statine, DCC/ HOBt, $50.7 \%$ for steps (a)-(c). 


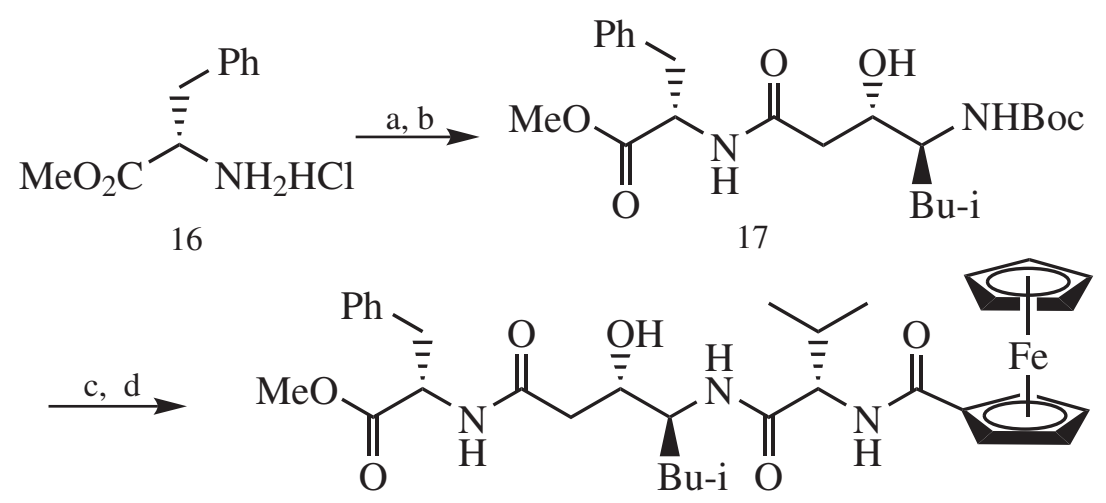

11

Scheme 2. Synthesis of compound 11. Reagents and conditions: (a) NMM/DCM, (b) N-Boc-statine, DCC/HOBt; (b) 4 mol L-1 $\mathrm{HCl} / \mathrm{dioxane}$; (c) NMM/ DCM; (d) FcCO-Val-OH, DCC/HOBt, $27.6 \%$ for steps (a)-(d).

structures. In the mass spectra they give peaks corresponding to the molecular ion. In the case of compounds 1-10, an intense fragment ion was observed at $\mathrm{m} / \mathrm{z}, 199$ and is due the $\left[\mathrm{FcCH}_{2}\right]^{+}$cation. Fragment ions corresponding to $[\mathrm{M}-65]^{+}$were also noted. This corresponds to loss of the unsubstituted $\left(\eta^{5}-\mathrm{C}_{5} \mathrm{H}_{5}\right)$ ring.

For compounds 1-10, their ${ }^{1} \mathrm{H}$ NMR spectra are characteristic: the ferrocenyl substituent gives rise to a fiveproton singlet for the unsubstituted cyclopentadienyl ring and a multiplet for the monosubstituted ring. The protons of the methylene unit adjacent to the ferrocene moiety appear as a doublet often overlapping with the singlet of the $\left(\eta^{5}-\mathrm{C}_{5} \mathrm{H}_{5}\right)$ ring.

In compound 11, the $\left(\eta^{5}-\mathrm{C}_{5} \mathrm{H}_{5}\right)$ ring appears as a singlet in ${ }^{1} \mathrm{H}$ NMR spectrum at $\delta 4.21$ whereas the meta and ortho protons on the $\left(\eta^{5}-\mathrm{C}_{5} \mathrm{H}_{4}\right)$ ring are present at $\delta 4.27$ and 4.71, respectively. The characteristic bands of the ferrocenyl group in the IR spectra of the compound $\mathbf{2}$ appear at 3091, $1440,1277,1175,1041$ and $744 \mathrm{~cm}^{-1}$.

All the target compounds were screened against the tested bacteria (Aspergillus oryzae) and the results of their biological activity are shown in Table 1 . Replacing valine with leucine, phenylalanine or 4-nitro-phenylalanine residue renders lower activity (4 vs $\mathbf{1}, \mathbf{2}, \mathbf{6}$, Table 1 ). This might suggest that the valine residue is necessary. Truncation of the $\mathrm{C}$-terminus by removal of the $\mathrm{C}$-terminal amino acid residue resulted in some loss of potency $(\mathbf{1}, \mathbf{2}, \mathbf{4}$, $\mathbf{7}$ vs $\mathbf{9}$, Table 1). Compound 8 was found to have an activity of $61.2 \%$ inhibition and higher than compound 4 . This might indicate that amino acid residue of $\mathrm{N}$-terminus is very important. Compound $\mathbf{3}$ and $\mathbf{5}$ having the $R$-configuration, and as expected they have no activity. Replacing Boc group with FcCO-Val residue leads to inhibitor $\mathbf{1 1}$ with $37.4 \%$ inhibition. Interestingly, bis-statine based compound $\mathbf{1 0}$ has an activity of $68.3 \%$ inhibition and higher than others.

\section{Conclusions}

In conclusion, a novel series of statine derivatives with short peptides containing ferrocenyl moiety have been prepared. All the compounds were characterized by elemental analyses, MS, ${ }^{1} \mathrm{H}$ NMR and IR spectra. The results of bioassay showed that compounds $\mathbf{4 ,} \mathbf{8}$ and $\mathbf{1 1}$ might serve as a starting point.

\section{Acknowledgements}

The authors thank the National Natural Science Foundation of China (No. 20572085, 20472067) for financial support.

\section{Supplementary Information}

Supplementary data are available free of charge at http:// jbcs.sbq.org.br, as PDF file.

\section{References}

1. Abdel-Meguid, S. S.; Med. Res. Rev. 1993, 13, 731.

2. Dunn, B. M.; Chem. Rev. 2002, 102, 4431.

3. Matsubara, H. \& Feder, J. In The Enzymes; Boyer, P. D., eds.; $3^{\text {rd }}$ Ed., Academic Press: New York, 1994, vol. 3, pp. 721.

4. Umezawa, H.; Aoyagi, T.; Morishima, H.; Matzuzak, M.; Hamada, M.; Takeuchi, T.; J. Antibiot. 1970, 23, 259; Rich, D. H.; J. Med. Chem 1985, 28, 263; Leung, D.; Abbenante, G.; Fairlie, D. P.; J. Med. Chem. 2000, 43, 305.

5. Fehrentz, J. A.; Chomier, B.; Bignon, E.; Venaud, S.; Chermann, J. C.; Nisato, D.; Biochem. Biophys. Res. Commun. 1992, 188, 873.

6. Dombrowski, K. E.; Baldwin, W.; Sheats, J. E.; J. Organomet. Chem. 1985, 302, 281. 
7. Top, S.; Vessières, A.; Cabestaing, C.; Laios, I.; Leclercq, G.; Provot, C.; Jaouen, G.; J. Organomet. Chem. 2001, 637, 500.

8. Biot, C.; Delhaes, L.; Abessolo, H.; Domarle, O.; Maciejewski, L. A.; Mortuaire, M.; Delcourt, P.; Deloron, P.; Camus, D.; Dive, D.; Brocard, J. S.; J. Organomet. Chem. 1999, 589, 59.

9. Silva, B. V.; Ribeiro, N. M.; Pinto, A. C.; Vargas, M. D.; Dias, L. C.; J. Braz. Chem. Soc. 2008, 19, 1244.

10. Edwards, E. I.; Epton, R.; Marr, G.; J. Organomet. Chem. 1976, 107, 351.

11. Edwards, E. I.; Epton, R.; Marr, G.; J. Organomet. Chem. 1976, 122, C49.

12. Biot, C.; Delhaes, L.; Abessolo, H.; Domarle, O.; Maciejewski, L. A.; Mortuaire, M.; Delcourt, P.; Deloron, P.; Camus, D.; Dive, D.; Brocard, J. S.; J. Organomet. Chem. 1999, 589, 59; Huang, R. Q.; Wang, Q. M.; J. Organomet. Chem. 2001, 637, 94.
13. Rich, D. H.; Sun, E. T.; Boparai, A. S.; J. Org. Chem. 1978, 43, 3624; Rich, D. H.; Sun, E. T.; J. Med. Chem. 1980, 23, 27.

14. Kelly, P. D.; Prêtre, A.; Devoy, S.; O’Rielly, I.; Devery, R.; Goel, A.; Gallagher, J. F.; Lough, A. J.; Kenny, P. T. M.; J. Organomet. Chem. 2007, 692, 1327.

15. Gu, L. J.; Liu, F. L.; Li, X. G.; Gao, B.; Yang, B. Q.; Synth. Commun. accepted manuscript.

16. Guo, H. Z.; Ma, L. Z.; Jiangsu Agr. Sci. 2007, 4, 211.

17. Abbreviations: N-Boc-AHPPA, (3S, 4S)- (tertButoxycarbonylamino)-3-hydroxy-5-phenyl-pentanoic acid; AHPPA, (3S, 4S)-4-amino-3-hydroxy-5-phenyl-pentanoic acid; Nph, 4-nitro-phenylalanine.

Received: January 16, 2009 Web Release Date: October 16, 2009 


\section{Synthesis and Biological Activity of Novel Statine Derivatives Containing Ferrocenyl Moiety}

\section{Lijun Gu, Bingqin Yang* and Feilong Liu}

Key Laboratory of Synthetic and Natural Functional Molecule Chemistry (Ministry of Education), Department of Chemistry, Northwest University, Xi'an 710069, People's Republic of China

\section{Boc-Leu-NH- $\mathrm{CH}_{2} \mathrm{FC} \mathrm{I4}$}

Yellow sticky oil, yield $73.5 \%,[\alpha]_{\mathrm{D}}{ }^{20}=-1.8^{\circ}(c 0.11$, $\mathrm{MeOH}$ ); IR (KBr): 3313, 3093, 2967, 2872, 1655, 1520, 1293 and $1168 \mathrm{~cm}^{-1} .{ }^{1} \mathrm{H} \mathrm{NMR}\left(\mathrm{CDCl}_{3}, 400 \mathrm{MHz}\right): \delta 0.93-$ $0.98\left\{\mathrm{~m}, 6 \mathrm{H},\left(\mathrm{CH}_{3}\right)_{2} \mathrm{CH}\right\}, 1.05-1.09\left(\mathrm{~m}, 1 \mathrm{H}, \mathrm{CHMe}_{2}\right), 1.44$ $\left\{\mathrm{s}, 9 \mathrm{H},\left(\mathrm{CH}_{3}\right)_{3}\right\}, 1.65-1.76\left(\mathrm{~m}, 2 \mathrm{H}, \mathrm{CH}_{2} \mathrm{CHMe}_{2}\right), 3.72(\mathrm{~d}$, $J=0.8 \mathrm{~Hz}, 1 \mathrm{H}, \mathrm{CHCO}), 4.11-4.16\left(\mathrm{~m}, 11 \mathrm{H}, \mathrm{C}_{5} \mathrm{H}_{4}, \mathrm{C}_{5} \mathrm{H}_{5}\right.$, $\mathrm{CH}_{2} \mathrm{~N}$ ), 4.87 (br, 1H, NH), 6.38 (br, 1H, NH). Anal. Calcd for $\mathrm{C}_{22} \mathrm{H}_{32} \mathrm{FeN}_{2} \mathrm{O}_{3}$ : C 61.69, H 7.53, N 6.54; found C 61.81, H 7.42, N 6.59.

\section{Boc-Phe-NH-CH Fc 17}

Yellow sticky oil, yield $71.4 \%,[\alpha]_{\mathrm{D}}{ }^{20}=+3.1^{\circ}(c 0.16$, $\mathrm{MeOH}$ ); IR (KBr): 3415, 3004, 2926, 1711, 1516, 1419, 1362, 1222, 1031, 903 and $531 \mathrm{~cm}^{-1} .{ }^{1} \mathrm{H} \mathrm{NMR}\left(\mathrm{CDCl}_{3}\right.$, $400 \mathrm{MHz}): \delta 1.39\left\{\mathrm{~s}, 9 \mathrm{H},\left(\mathrm{CH}_{3}\right)_{3}\right\}, 3.05(\mathrm{~d}, J=8 \mathrm{~Hz}, 2 \mathrm{H}$, $\left.\mathrm{CH}_{2} \mathrm{Ph}\right), 4.03-4.19\left(\mathrm{~m}, 11 \mathrm{H}, \mathrm{C}_{5} \mathrm{H}_{4}, \mathrm{C}_{5} \mathrm{H}_{5}, \mathrm{CH}_{2} \mathrm{~N}\right), 4.32$ (br, $1 \mathrm{H}, \mathrm{CHCO}), 5.10$ (s, 1H, NH), 6.10 (s, 1H, NH), 7.18-7.30 (m, 5H, ArH). Anal. Calcd for $\mathrm{C}_{25} \mathrm{H}_{30} \mathrm{FeN}_{2} \mathrm{O}_{3}: \mathrm{C} 64.94, \mathrm{H}$ 6.54, N 6.06; found C 65.17, H 6.83, N 6.21.

\section{Boc-Val- $\mathrm{NH}-\mathrm{CH}_{2} \mathrm{FC} \mathbf{1 8}$}

Yellow sticky oil, yield $77.2 \%,[\alpha]_{\mathrm{D}}{ }^{20}=-0.6^{\circ}$ (c 0.51, $\mathrm{MeOH}$ ); IR (KBr): 3311, 3092, 2967, 2874, 1656, 1520, 1244 and $1169 \mathrm{~cm}^{-1} .{ }^{1} \mathrm{H} \mathrm{NMR}\left(\mathrm{CDCl}_{3}, 400 \mathrm{MHz}\right): \delta 0.96$ (q, J=0.8Hz, 6H, $\left.\left(\mathrm{CH}_{3}\right)_{2} \mathrm{CH}\right), 1.17-1.25\left(\mathrm{~m}, 1 \mathrm{H}, \mathrm{CHMe}_{2}\right)$, $1.44\left\{\mathrm{~s}, 9 \mathrm{H},\left(\mathrm{CH}_{3}\right)_{3}\right\}, 3.87(\mathrm{t}, J=0.4 \mathrm{~Hz}, 1 \mathrm{H}, \mathrm{CHCO}), 4.13-$ 4.17 ( $\left.\mathrm{m}, 11 \mathrm{H}, \mathrm{C}_{5} \mathrm{H}_{4}, \mathrm{C}_{5} \mathrm{H}_{5}, \mathrm{CH}_{2} \mathrm{~N}\right), 5.00$ (br, 1H, NH), 6.15 (br, 1H, NH). Anal. Calcd for $\mathrm{C}_{21} \mathrm{H}_{30} \mathrm{FeN}_{2} \mathrm{O}_{3}: \mathrm{C} 60.88, \mathrm{H}$ 7.30, N 6.76; found C 60.75, H 7.18, N 6.55.

\section{Boc- $\mathrm{Nph}-\mathrm{NH}-\mathrm{CH}_{2} \mathrm{Fc} 19$}

Yellow sticky oil, yield $65.5 \%,[\alpha]_{\mathrm{D}}^{20}=+0.97^{\circ}(\mathrm{c} 0.23$, $\mathrm{MeOH}$ ); IR (KBr): 3305, 3086, 2931, 2868, 1656, 1602, $1521,1452,1345,1248,1165,1021,854$ and $486 \mathrm{~cm}^{-1}$. ${ }^{1} \mathrm{H}$ NMR $\left(\mathrm{CDCl}_{3}, 400 \mathrm{MHz}\right): \delta 1.39\left\{\mathrm{~s}, 9 \mathrm{H},\left(\mathrm{CH}_{3}\right)_{3}\right\}, 3.11$

*e-mail: gulijun2005@126.com (q, J=0.8 Hz, 2H, CH $2 \mathrm{Ph}), 3.21-3.26$ (m, 1H, CHCO), 4.05-4.19 (m, 11H, $\left.\mathrm{C}_{5} \mathrm{H}_{4}, \mathrm{C}_{5} \mathrm{H}_{5}, \mathrm{CH}_{2} \mathrm{~N}\right), 5.08$ (d, J=5.6 Hz, 1H, NH), 6.07 (d, J=6.6 Hz, 1H, NH), 7.33 (q, J=8.8 Hz, 2H, ArH), 8.15 (q, J=6.4 Hz, 2H, ArH). Anal. Calcd for $\mathrm{C}_{25} \mathrm{H}_{29} \mathrm{FeN}_{3} \mathrm{O}_{5}$ : C 59.18, H 5.76, N 8.28; found C 59.20, H 5.83, N 8.24.

\section{Boc-Val-Phe- $\mathrm{NH}-\mathrm{CH}_{2} \mathrm{Fc} 20$}

Yellow sticky oil, yield 71.4\%, $[\alpha]_{\mathrm{D}}{ }^{20}=-9.4^{\circ}($ c 0.16, $\mathrm{MeOH}$ ); IR (KBr): 3422, 2932, 1663, 1497, 1439, 1390, 1253, 1101 and $700 \mathrm{~cm}^{-1}$. ${ }^{1} \mathrm{H} \mathrm{NMR}\left(\mathrm{CDCl}_{3}, 400 \mathrm{MHz}\right): \delta$ 0.79-0.91 $\left\{\mathrm{m}, 6 \mathrm{H},\left(\mathrm{CH}_{3}\right)_{2} \mathrm{CH}\right\}, 1.38\left\{\mathrm{~s}, 9 \mathrm{H},\left(\mathrm{CH}_{3}\right)_{3}\right\}, 1.43-$ 1.45 (m, 1H, CHMe $), 2.99$ (m, 2H, CH $2 \mathrm{Ph}), 3.04$ (t, J=1.6 $\mathrm{Hz}, 1 \mathrm{H}, \mathrm{CHCO}), 3.87$ (t, J=6 Hz, 1H, CHCO), 4.04-4.17 (m, $\left.11 \mathrm{H}, \mathrm{C}_{5} \mathrm{H}_{4}, \mathrm{C}_{5} \mathrm{H}_{5}, \mathrm{CH}_{2} \mathrm{~N}\right), 4.62(\mathrm{~d}, J=6.4 \mathrm{~Hz}, 1 \mathrm{H}, \mathrm{NH})$, 4.81 (br, $1 \mathrm{H}, \mathrm{NH}) ; 6.17$ (br, $1 \mathrm{H}, \mathrm{NH}) ; 7.28-7.16$ (m, 5H, ArH). Anal. Calcd for $\mathrm{C}_{30} \mathrm{H}_{39} \mathrm{FeN}_{3} \mathrm{O}_{4}: \mathrm{C} 64.17, \mathrm{H} 7.00, \mathrm{~N}$ 7.48; found C 64.31, H 7.14, N 7.36.

\section{Boc-Statine-Leu-NH-CH $\mathrm{FC}_{2} \mathrm{I}$}

Yellow solid, yield $49.2 \%$, m.p. $71-72{ }^{\circ} \mathrm{C},[\alpha]_{\mathrm{D}}{ }^{20}=-41^{\circ}$ (c 0.10, MeOH ); IR (KBr): 3325, 3092, 2957, 2930, 2870, $1689,1645,1535,1465,1366,1248,1169,1045,881$ and $644 \mathrm{~cm}^{-1} .{ }^{1} \mathrm{H} \mathrm{NMR}\left(\mathrm{CDCl}_{3}, 400 \mathrm{MHz}\right): \delta 0.75-0.98$ $\left\{\mathrm{m}, 12 \mathrm{H},\left(\mathrm{CH}_{3}\right)_{2} \mathrm{CH},\left(\mathrm{CH}_{3}\right)_{2} \mathrm{CHCH}_{2}\right\}, 1.23-1.49(\mathrm{~m}, 6 \mathrm{H}$, $\left.\mathrm{CH}_{2} \mathrm{CHMe}_{2}, \mathrm{CH}_{2} \mathrm{CHMe}_{2}\right), 1.53\left\{\mathrm{~s}, 9 \mathrm{H},\left(\mathrm{CH}_{3}\right)_{3}\right\}, 2.23-2.40$ (m, 2H, $\mathrm{CH}_{2} \mathrm{CO}$ ), 3.64-3.74 (m, 1H, NCH-Bu-i), 3.87-3.95 (m, 2H, NCHCO, CH-O), 3.97-4.09 (m, 11H, $\mathrm{C}_{5} \mathrm{H}_{4}, \mathrm{C}_{5} \mathrm{H}_{5}$, $\mathrm{CH}_{2} \mathrm{~N}$ ), 4.30 (q, $\left.1 \mathrm{H}, J=8 \mathrm{~Hz}, \mathrm{NH}\right), 4.65$ (d, $J=8 \mathrm{~Hz}, 1 \mathrm{H}$, $\mathrm{NH}), 6.32$ (d, $J=8 \mathrm{~Hz}, 1 \mathrm{H}, \mathrm{NH})$. MALDI-TOF MS: $\mathrm{m} / \mathrm{z}$ $=586[\mathrm{M}+1]^{+}$. Anal. Calcd for $\mathrm{C}_{30} \mathrm{H}_{47} \mathrm{FeN}_{3} \mathrm{O}_{5}: \mathrm{C} 61.53, \mathrm{H}$ 8.09, N 7.18; found C 61.64, H

\section{Boc-Statine-Phe-NH- $\mathrm{CH}_{2} \mathrm{FC} 2$}

Yellow solid, yield 53.6\%, m.p. $79-81^{\circ} \mathrm{C},[\alpha]_{\mathrm{D}}{ }^{20}=-44^{\circ}$ (c 0.21, MeOH ); IR (KBr): 3310, 3088, 3031, 2956, 2929, 2869, 1688, 1643, 1532, 1452, 1365, 1248, 1168, 1044, 817 and $744 \mathrm{~cm}^{-1} .{ }^{1} \mathrm{H} \mathrm{NMR}\left(\mathrm{CDCl}_{3}, 400 \mathrm{MHz}\right): \delta 0.75-0.86$ 
$\left\{\mathrm{m}, 6 \mathrm{H},\left(\mathrm{CH}_{3}\right)_{2} \mathrm{CHCH}_{2}\right\}, 1.33-1.43\left(\mathrm{~m}, 3 \mathrm{H}, \mathrm{CH}_{2} \mathrm{CHMe}_{2}\right)$, $1.56\left\{\mathrm{~s}, 9 \mathrm{H},\left(\mathrm{CH}_{3}\right)_{3}\right\}, 2.17-2.36\left(\mathrm{~m}, 2 \mathrm{H}, \mathrm{CH}_{2} \mathrm{CO}\right), 2.90-3.07$ (m, 2H, $\left.\mathrm{CH}_{2} \mathrm{Ph}\right), 3.45-3.57(\mathrm{~m}, 1 \mathrm{H}, \mathrm{NCH}-\mathrm{Bu}-\mathrm{i}), 3.84-3.93$ (m, 2H, NCHCO, CH-O), 3.95-4.04(m, $11 \mathrm{H}, \mathrm{C}_{5} \mathrm{H}_{4}, \mathrm{C}_{5} \mathrm{H}_{5}$, $\left.\mathrm{CH}_{2} \mathrm{~N}\right), 4.62(\mathrm{~d}, J=9.6 \mathrm{~Hz}, 1 \mathrm{H}, \mathrm{NH}), 5.98(\mathrm{br}, 1 \mathrm{H}, \mathrm{NH}), 6.46$ (d, $J=8 \mathrm{~Hz}, 1 \mathrm{H}, \mathrm{NH}), 7.10-7.20$ (m, 5H, ArH). MALDITOF MS: $m / z=619\left(\mathrm{M}^{+}\right)$. Anal. Calcd for $\mathrm{C}_{33} \mathrm{H}_{45} \mathrm{FeN}_{3} \mathrm{O}_{5}$ : C 63.97, H 7.32, N 6.78; found C 63.74, H 7.45, N 6.62.

\section{Boc-(3R, 4S)-Statine-Phe-NH- $\mathrm{CH}_{2} \mathrm{Fc}_{3}$}

Yellow solid, yield $53.6 \%$, m.p. $85-87{ }^{\circ} \mathrm{C},[\alpha]_{\mathrm{D}}{ }^{20}=$ $-30.7^{\circ}$ ( c 0.17, MeOH ); IR (KBr): 3333, 3088, 2953, 2930, 2853, 1681, 1633, 1532, 1449, 1368, 1248, 1169,698 and $670 \mathrm{~cm}^{-1} .{ }^{1} \mathrm{H}$ NMR $\left(\mathrm{CDCl}_{3}, 400 \mathrm{MHz}\right)$ : $\delta 0.85-0.93\left(\mathrm{~m}, 6 \mathrm{H},\left(\mathrm{CH}_{3}\right)_{2} \mathrm{CHCH}_{2}\right), 1.31-1.33(\mathrm{~m}, 3 \mathrm{H}$, $\left.\mathrm{CH}_{2} \mathrm{CHMe}_{2}\right), 1.57\left\{\mathrm{~s}, 9 \mathrm{H},\left(\mathrm{CH}_{3}\right)_{3}\right\}, 2.25-2.43(\mathrm{~m}, 2 \mathrm{H}$, $\left.\mathrm{CH}_{2} \mathrm{CO}\right), 3.00-3.14\left(\mathrm{~m}, 2 \mathrm{H}, \mathrm{CH}_{2} \mathrm{Ph}\right), 3.52-3.59(\mathrm{~m}, 1 \mathrm{H}$, NCH-Bu-i), 3.73-3.91 (m, 2H, NCHCO, CH-O), 4.03$4.10\left(\mathrm{~m}, 11 \mathrm{H}, \mathrm{C}_{5} \mathrm{H}_{4}, \mathrm{C}_{5} \mathrm{H}_{5}, \mathrm{CH}_{2} \mathrm{~N}\right), 5.99(\mathrm{br}, 1 \mathrm{H}, \mathrm{NH})$, $6.38(\mathrm{~d}, J=7.6 \mathrm{~Hz}, 1 \mathrm{H}, \mathrm{NH}), 6.47(\mathrm{~d}, J=8.0 \mathrm{~Hz}, 1 \mathrm{H}, \mathrm{NH})$, 7.17-7.24 (m, 5H, ArH). MALDI-TOF MS: $m / z=619$ $\left(\mathrm{M}^{+}\right)$. Anal. Calcd for $\mathrm{C}_{33} \mathrm{H}_{45} \mathrm{FeN}_{3} \mathrm{O}_{5}: \mathrm{C} 63.97, \mathrm{H} 7.32, \mathrm{~N}$ 6.78; found C 63.81, H 7.19, N 6.90.

\section{Boc-Statine-Val-NH- $\mathrm{CH}_{2} \mathrm{Fc} 4$}

Yellow solid, yield $50.7 \%$, m.p. $86-88{ }^{\circ} \mathrm{C}$ [lit. ${ }^{[15]} 86-$ $\left.88^{\circ} \mathrm{C}\right],[\alpha]_{\mathrm{D}}^{20}=-41.4^{\circ}$ ( $\left.0.23, \mathrm{MeOH}\right)$; IR (KBr): 3326, 3095, 2929, 2851, 1688, 1631, 1533, 1444, 1367, 1246, 1167, 1046, 821, 641 and $482 \mathrm{~cm}^{-1} .{ }^{1} \mathrm{H}$ NMR $\left(\mathrm{CDCl}_{3}, 400\right.$ $\mathrm{MHz}): \delta 0.82-0.95\left\{\mathrm{~m}, 12 \mathrm{H},\left(\mathrm{CH}_{3}\right)_{2} \mathrm{CH},\left(\mathrm{CH}_{3}\right)_{2} \mathrm{CHCH}_{2}\right\}$, 1.20-1.48 (m, 4H, $\left.\mathrm{CHMe}_{2}, \mathrm{CH}_{2} \mathrm{CHMe}_{2}\right), 1.59\{\mathrm{~s}, 9 \mathrm{H}$, $\left.\left(\mathrm{CH}_{3}\right)_{3}\right\}, 2.32-2.43\left(\mathrm{~m}, 2 \mathrm{H}, \mathrm{CH}_{2} \mathrm{CO}\right), 3.48(\mathrm{q}, 1 \mathrm{H}, \mathrm{J}=0.4 \mathrm{~Hz}$, NCH-Bu-i), 3.83-3.95 (m, 2H, NCHCO, CH-O), 4.12-4.21 $\left(\mathrm{m}, 11 \mathrm{H}, \mathrm{C}_{5} \mathrm{H}_{4}, \mathrm{C}_{5} \mathrm{H}_{5}, \mathrm{CH}_{2} \mathrm{~N}\right), 4.73(\mathrm{~d}, J=12 \mathrm{~Hz}, 1 \mathrm{H}, \mathrm{NH})$, 6.24 (br, 1H, NH), 6.51 (d, $J=8 \mathrm{~Hz}, 1 \mathrm{H}, \mathrm{NH})$. MALDITOF MS: $m / z=571\left(\mathrm{M}^{+}\right)$. Anal. Calcd for $\mathrm{C}_{29} \mathrm{H}_{45} \mathrm{FeN}_{3} \mathrm{O}_{5}$ : C 60.94, H 7.94, N 7.35; found C 60.85, H 7.88, N 7.29.

\section{Boc-(3R, 4S)-Statine-Val-NH-CH $\mathrm{Fc}_{2} 5$}

Yellow solid, yield 54.3\%, m.p. $89-91{ }^{\circ} \mathrm{C},[\alpha]_{\mathrm{D}}{ }^{20}=-27^{\circ}$ (c 0.21, MeOH ); IR (KBr): 3326, 3091, 2954, 2932, 2871, $1685,1626,1538,1440,1367,1277,1175,1041,744$ and $483 \mathrm{~cm}^{-1} .{ }^{1} \mathrm{H}$ NMR $\left(\mathrm{CDCl}_{3}, 400 \mathrm{MHz}\right): \delta 0.80-0.95\{\mathrm{~m}$, $\left.12 \mathrm{H},\left(\mathrm{CH}_{3}\right)_{2} \mathrm{CH},\left(\mathrm{CH}_{3}\right)_{2} \mathrm{CHCH}_{2}\right\}, 1.44\left\{\mathrm{~s}, 9 \mathrm{H},\left(\mathrm{CH}_{3}\right)_{3}\right\}$, 1.50-1.68 $\left\{\mathrm{m}, 4 \mathrm{H}, \mathrm{CHMe}_{2}, \mathrm{CH}_{2} \mathrm{CHMe}_{2}\right\}, 2.33-2.40(\mathrm{~m}$, $2 \mathrm{H}, \mathrm{CH}_{2} \mathrm{CO}$ ), 3.46-3.56 (m, 1H, NCH-Bu-i), 3.71-3.95 (m, 2H, NCHCO, CH-O), 4.02-4.18 (m, $11 \mathrm{H}, \mathrm{C}_{5} \mathrm{H}_{4}, \mathrm{C}_{5} \mathrm{H}_{5}$, $\left.\mathrm{CH}_{2} \mathrm{~N}\right), 4.73$ (d, J=9.6 Hz, 1H, NH), 6.22(br, 1H, NH), $6.51(\mathrm{~d}, J=8.4 \mathrm{~Hz}, 1 \mathrm{H}, \mathrm{NH})$. MALDI-TOF MS: $\mathrm{m} / z=572$ $[\mathrm{M}+\mathrm{H}]^{+}$. Anal. Calcd for $\mathrm{C}_{29} \mathrm{H}_{45} \mathrm{FeN}_{3} \mathrm{O}_{5}: \mathrm{C} 60.94, \mathrm{H}$ 7.94, $\mathrm{N}$ 7.35; found $\mathrm{C}$ 60.73, H 8.01, N 7.17.

\section{Boc-Statine-Nph- $\mathrm{NH}-\mathrm{CH}_{2} \mathrm{Fc} 6$}

Yellow solid, yield $40.5 \%$, m.p. $96-98^{\circ} \mathrm{C},[\alpha]_{\mathrm{D}}{ }^{20}=-39^{\circ}$ (c 0.15, MeOH ); IR (KBr): 3319, 3077, 2956, 1689, 1647, $1522,1445,1345,1167,1046,845$ and $486 \mathrm{~cm}^{-1} .{ }^{1} \mathrm{H} \mathrm{NMR}$ $\left(\mathrm{CDCl}_{3}, 400 \mathrm{MHz}\right): \delta 0.92\left\{\mathrm{q}, J=1.2 \mathrm{~Hz}, 6 \mathrm{H},\left(\mathrm{CH}_{3}\right)_{2} \mathrm{CH}\right\}$, 1.07-1.11 (m, 1H, CHMe 2$), 1.44\left\{\mathrm{~s}, 9 \mathrm{H},\left(\mathrm{CH}_{3}\right)_{3}\right\}, 1.56-1.71$ (m, $2 \mathrm{H}, \mathrm{CH}_{2} \mathrm{CHMe}_{2}$ ), 2.31-2.40 (m, 2H, $\mathrm{CH}_{2} \mathrm{CO}$ ), 3.14-3.22 (m, 2H, $\mathrm{CH}_{2}-\mathrm{Ar}$ ), 3.43-3.51 (m, 1H, NCH-Bu-i), 3.71-3.95 (m, 2H, NCHCO, CH-O), 4.07-4.16 (m, 11H, $\mathrm{C}_{5} \mathrm{H}_{4}, \mathrm{C}_{5} \mathrm{H}_{5}$, $\left.\mathrm{CH}_{2} \mathrm{~N}\right), 4.71(\mathrm{~d}, J=4.4 \mathrm{~Hz}, 1 \mathrm{H}, \mathrm{NH}), 6.42(\mathrm{~d}, J=4.4 \mathrm{~Hz}$, $1 \mathrm{H}, \mathrm{NH}), 6.69$ (d, J=8.0 Hz, 1H, NH), 7.33 (q, J=5.2 Hz, $2 \mathrm{H}, \mathrm{ArH}), 8.08$ (q, J=8.4 Hz, 2H, ArH). MALDI-TOF MS: $m / z=664\left(\mathrm{M}^{+}\right)$. Anal. Calcd for $\mathrm{C}_{33} \mathrm{H}_{44} \mathrm{FeN}_{4} \mathrm{O}_{7}: \mathrm{C} 59.64, \mathrm{H}$ 6.67, N 8.43; found C 59.67, H 6.71, N 8.53.

\section{Boc-Statine-Val-Phe-NH- $\mathrm{CH}_{2} \mathrm{Fc} 7$}

Yellow solid, yield $30.2 \%$, m.p. $166-168{ }^{\circ} \mathrm{C},[\alpha]_{\mathrm{D}}{ }^{20}=$ $-53^{\circ}$ ( c 0.10, MeOH ); IR (KBr): 3422, 2932, 1663, 1497 , 1439, 1390, 1253, 1101 and $700 \mathrm{~cm}^{-1} .{ }^{1} \mathrm{H} \mathrm{NMR}\left(\mathrm{CDCl}_{3}, 400\right.$ $\mathrm{MHz}): \delta 0.86-0.99\left\{\mathrm{~m}, 12 \mathrm{H},\left(\mathrm{CH}_{3}\right)_{2} \mathrm{CH},\left(\mathrm{CH}_{3}\right)_{2} \mathrm{CHCH}_{2}\right\}$, 1.28-1.38 (m, 2H, CHMe, $\left.\mathrm{CHMe}_{2}\right), 1.44\left\{\mathrm{~s}, 9 \mathrm{H},\left(\mathrm{CH}_{3}\right)_{3}\right\}$, 1.50-1.69 \{ m, 2H, $\left.\mathrm{CH}_{2}-\mathrm{Pr}-\mathrm{i}\right\}, 2.49-2.62\left(\mathrm{~m}, 2 \mathrm{H}, \mathrm{CH}_{2} \mathrm{CO}\right)$, 3.05-3.17 (m, 2H, $\left.\mathrm{CH}_{2} \mathrm{Ph}, \mathrm{NCH}-\mathrm{Bu}-\mathrm{i}\right), 3.50$ (t, $J=3.6 \mathrm{~Hz}$, 1H, NCHCO), 3.89 (br, 1H, CH-O), 4.00-4.17 (m, 12H, $\left.\mathrm{NCHCO}, \mathrm{C}_{5} \mathrm{H}_{4}, \mathrm{C}_{5} \mathrm{H}_{5}, \mathrm{CH}_{2} \mathrm{~N}\right), 4.86(\mathrm{~d}, J=7.6 \mathrm{~Hz}, 1 \mathrm{H}, \mathrm{NH})$, 5.04 (d, J=8.4 Hz, 1H, NH), 5.51 (br, 1H, NH), 6.38 (br, $1 \mathrm{H}, \mathrm{NH}), 7.30-7.10$ (m, 5H, ArH). MALDI-TOF MS: $\mathrm{m} / \mathrm{z}$ $=718\left(\mathrm{M}^{+}\right)$. Anal. Calcd for $\mathrm{C}_{38} \mathrm{H}_{54} \mathrm{FeN}_{4} \mathrm{O}_{6}: \mathrm{C} 63.50, \mathrm{H} 7.57$, N 7.80; found C 63.62, H 7.66, N 7.65.

\section{Boc-Val-Statine-Val- $\mathrm{NH}-\mathrm{CH}_{2} \mathrm{Fc} 8$}

Yellow solid, yield $32.4 \%$, m.p. $142-144{ }^{\circ} \mathrm{C},[\alpha]_{\mathrm{D}}{ }^{20}=$ $-49^{\circ}$ ( c 0.10, $\mathrm{MeOH}$ ); IR (KBr): 3326, 3086, 2929, 2850, $1687,1627,1573,1534,1367,1245,1167,1087$ and 642 $\mathrm{cm}^{-1} .{ }^{1} \mathrm{H}$ NMR $\left(\mathrm{CDCl}_{3}, 400 \mathrm{MHz}\right): \delta 0.81-0.96\{\mathrm{~m}, 18 \mathrm{H}$, $\left.\left(\mathrm{CH}_{3}\right)_{2} \mathrm{CH},\left(\mathrm{CH}_{3}\right)_{2} \mathrm{CH},\left(\mathrm{CH}_{3}\right)_{2} \mathrm{CHCH}_{2}\right\}, 1.04-1.43(\mathrm{~m}, 3 \mathrm{H}$, $\left.\mathrm{CHMe}_{2}, \mathrm{CHMe}_{2}, \mathrm{CHMe}_{2},\right), 1.57\left\{\mathrm{~s}, 9 \mathrm{H},\left(\mathrm{CH}_{3}\right)_{3}\right\}, 1.61-1.70$ $\left(\mathrm{CH}_{2}-\mathrm{Pr}-\mathrm{i}\right), 2.33-2.38\left(2 \mathrm{H}, \mathrm{CH}_{2} \mathrm{CO}\right), 3.45-3.56(\mathrm{~m}, 2 \mathrm{H}$, $\mathrm{NCH}-\mathrm{Bu}-\mathrm{i}, \mathrm{NCHCO}), 3.71-3.99$ (m, 2H, NCHCO, $\mathrm{CH}-\mathrm{O}$ ), 4.02-4.17 (m, $\left.11 \mathrm{H}, \mathrm{C}_{5} \mathrm{H}_{4}, \mathrm{C}_{5} \mathrm{H}_{5}, \mathrm{CH}_{2} \mathrm{~N}\right), 4.76(\mathrm{~d}, J=9.2 \mathrm{~Hz}$, $1 \mathrm{H}, \mathrm{NH}), 6.21$ (br, $1 \mathrm{H}, \mathrm{NH}), 6.51$ (d, $J=8.8 \mathrm{~Hz}, 1 \mathrm{H}, \mathrm{NH}$ ), $6.65(\mathrm{br}, 1 \mathrm{H}, \mathrm{NH})$. MALDI-TOF MS: $m / z=670\left(\mathrm{M}^{+}\right)$. Anal. Calcd for $\mathrm{C}_{34} \mathrm{H}_{54} \mathrm{FeN}_{4} \mathrm{O}_{6}: \mathrm{C} 60.89, \mathrm{H} 8.12, \mathrm{~N} 8.35$; found $\mathrm{C}$ 61.01, H 8.20, N 8.29.

\section{Boc-Statine- $\mathrm{NH}-\mathrm{CH}_{2} \mathrm{FC} 9$}

Yellow solid, yield $57.7 \%$, m.p. $109-111{ }^{\circ} \mathrm{C},[\alpha]_{\mathrm{D}}{ }^{20}=$ $-21^{\circ}$ (c 0.17, $\mathrm{MeOH}$ ); IR (KBr): 3327, 3095, 2929, 2850, $1688,1627,1534,1366,1246,1168,816,643$ and 482 $\mathrm{cm}^{-1}$. ${ }^{1} \mathrm{H}$ NMR $\left(\mathrm{CDCl}_{3}, 400 \mathrm{MHz}\right): \delta 0.88\{\mathrm{q}, J=0.4 \mathrm{~Hz}$, $\left.6 \mathrm{H},\left(\mathrm{CH}_{3}\right)_{2} \mathrm{CH}\right\}, 1.26-1.39$ (m, 3H, $\left.\mathrm{CH}_{2} \mathrm{CHMe}_{2}\right), 1.58\{\mathrm{~s}$, 
$\left.9 \mathrm{H},\left(\mathrm{CH}_{3}\right)_{3}\right\}, 2.28-2.39\left(\mathrm{~m}, 2 \mathrm{H}, \mathrm{CH}_{2} \mathrm{CO}\right), 3.39-3.56(\mathrm{~m}, 1 \mathrm{H}$, NCH-Bu-i), 3.95-3.98 (m, 1H, CH-O), 4.01-4.20 (m, 11H, $\left.\mathrm{C}_{5} \mathrm{H}_{4}, \mathrm{C}_{5} \mathrm{H}_{5}, \mathrm{CH}_{2} \mathrm{~N}\right), 4.72(\mathrm{~d}, J=9.6 \mathrm{~Hz}, 1 \mathrm{H}, \mathrm{NH}), 6.09$ (br, 1H). MALDI-TOF MS: $m / z=472\left(\mathbf{M}^{+}\right)$. Anal. Calcd for $\mathrm{C}_{24} \mathrm{H}_{36} \mathrm{FeN}_{2} \mathrm{O}_{4}: \mathrm{C} 61.02, \mathrm{H}$ 7.68, $\mathrm{N}$ 5.93; found C 61.44, $\mathrm{H} 7.65, \mathrm{~N} 6.01$.

\section{Boc-AHPPA-Statine-Val-NH- $\mathrm{CH}_{2} \mathrm{Fc} \mathbf{1 0}$}

Yellow solid, yield $20.3 \%$, m.p. $125-127{ }^{\circ} \mathrm{C}$ [lit. ${ }^{[15]}$ $\left.125-127{ }^{\circ} \mathrm{C}\right],[\alpha]_{\mathrm{D}}{ }^{20}=-62.6^{\circ}$ ( $\left.\mathrm{c} 0.10, \mathrm{MeOH}\right)$; IR $(\mathrm{KBr})$ : $3323,3088,2960,2928,1648,1534,1451,1366,1247$, $1167,1047,817,699$ and $483 \mathrm{~cm}^{-1} .{ }^{1} \mathrm{H}$ NMR $\left(\mathrm{CDCl}_{3}\right): \delta$ 0.82-0.98 $\left\{\mathrm{m}, 12 \mathrm{H},\left(\mathrm{CH}_{3}\right)_{2} \mathrm{CH},\left(\mathrm{CH}_{3}\right)_{2} \mathrm{CH}_{2} \mathrm{CH}\right\}, 1.33-1.38$ (m, 2H, CHMe, $\left.\mathrm{CHCH}_{2} \mathrm{Me}_{2}\right), 1.42\left\{\mathrm{~s}, 9 \mathrm{H},\left(\mathrm{CH}_{3}\right)_{3}\right\}, 1.54-$ $1.69\left(\mathrm{~m}, 2 \mathrm{H}, \mathrm{CHCH}_{2} \mathrm{Me}_{2}\right), 2.24-2.53\left(\mathrm{~m}, 4 \mathrm{H}, \mathrm{CH}_{2} \mathrm{CO}\right.$, $\mathrm{CH}_{2} \mathrm{CO}$ ), 2.91 (t, $\left.2 \mathrm{H}, J=2.8 \mathrm{~Hz}, \mathrm{CH}_{2} \mathrm{Ph}\right), 3.49-3.52(\mathrm{~m}, 1 \mathrm{H}$, NCHCO), 3.74-3.78 (m, 2H, CH-O, CH-O), 4.01-4.09 (m, $2 \mathrm{H}, \mathrm{NCHBn}, \mathrm{NCHBu}-\mathrm{i}), 4.12-4.26\left(\mathrm{~m}, 11 \mathrm{H}, \mathrm{C}_{5} \mathrm{H}_{4}, \mathrm{C}_{5} \mathrm{H}_{5}\right.$, $\left.\mathrm{CH}_{2} \mathrm{~N}\right), 4.97(\mathrm{~d}, 1 \mathrm{H}, J=8.8 \mathrm{~Hz}, \mathrm{NH}), 6.24$ (br, $1 \mathrm{H}, \mathrm{NH}$ ), 6.38 (br, 1H, NH), 6.76 (d, 1H, J=8.8 Hz, NH), 7.18-7.27
(m,5H, ArH). MALDI-TOF MS: $m / z=762\left(\mathrm{M}^{+}\right)$. Anal. Calcd for $\mathrm{C}_{40} \mathrm{H}_{58} \mathrm{FeN}_{4} \mathrm{O}_{7} \cdot \mathrm{H}_{2} \mathrm{O}: \mathrm{C} 61.53, \mathrm{H} 7.75, \mathrm{~N} 7.18$; found $\mathrm{C} 61.55, \mathrm{H} 7.78, \mathrm{~N} 7.21$.

\section{FcCO-Val-Statine-Phe-OMe 11}

Yellow solid, yield $27.6 \%$, m.p. $105-107{ }^{\circ} \mathrm{C},[\alpha]_{\mathrm{D}}{ }^{20}$ $=-50^{\circ}$ ( c 0.13, MeOH ); IR (KBr): 3447, 3097, 2925, 1774, 1678, 1488, 1367, 1260, 1055, 881, 746, 523 and $493 \mathrm{~cm}^{-1} .{ }^{1} \mathrm{H}$ NMR $\left(\mathrm{CDCl}_{3}, 400 \mathrm{MHz}\right): \delta$ 0.91-0.99 \{ m, $\left.12 \mathrm{H},\left(\mathrm{CH}_{3}\right)_{2} \mathrm{CH}_{2},\left(\mathrm{CH}_{3}\right)_{2} \mathrm{CH}_{2}\right\}, 1.39-1.42\left(\mathrm{~m}, 2 \mathrm{H}, \mathrm{CHMe}_{2}\right.$, $\mathrm{CHMe}_{2}$ ), 1.59-1.75 (m, 2H, $\left.\mathrm{CH}_{2}-\mathrm{Pr}-\mathrm{i}\right), 2.37-2.38$ (d, J=6.4 $\mathrm{Hz}, 2 \mathrm{H}, \mathrm{CH}_{2} \mathrm{CO}$ ), 3.01 (q, J=6.4 Hz, 2H, $\mathrm{CH}_{2} \mathrm{Ph}$ ), 3.10 (q, $J=5.6 \mathrm{~Hz}, 2 \mathrm{H}, \mathrm{NCH}-\mathrm{Bu}-\mathrm{i}), 3.73$ (s, $3 \mathrm{H}, \mathrm{CH}_{3} \mathrm{O}$ ), $4.02-4.12$ (br, 1H, NCHCO), 4.21 (s, 5H, Fc), 4.27 (d, J=2 Hz, 2H, FcCO), 4.71 (d, $J=1.2 \mathrm{~Hz}, 2 \mathrm{H}, \mathrm{FcCO}$ ), 4.79 (q, $J=6.4 \mathrm{~Hz}$, $1 \mathrm{H}, \mathrm{NH}), 5.98(\mathrm{~d}, J=7.6 \mathrm{~Hz}, 1 \mathrm{H}, \mathrm{NH}), 6.41(\mathrm{~d}, J=9.2 \mathrm{~Hz}$, 1H), 7.07-7.24 (m, 5H, ArH). MALDI-TOF MS: $\mathrm{m} / z=647$ $\left(\mathrm{M}^{+}\right)$. Anal. Calcd for $\mathrm{C}_{34} \mathrm{H}_{45} \mathrm{FeN}_{3} \mathrm{O}_{6}: \mathrm{C}$ 63.06, $\mathrm{H} 7.00, \mathrm{~N}$ 6.49; found $\mathrm{C} 63.23, \mathrm{H} 7.11, \mathrm{~N} 6.38$. 